\title{
Depression as a Mediator of Chronic Fatigue and Post-Traumatic Stress Symptoms in Middle East Respiratory Syndrome Survivors
}

\author{
So Hee Lee ${ }^{1}$, Hyoung-Shik Shin ${ }^{凶}$, Hye Yoon Park ${ }^{3}$, Jeong Lan Kim4, \\ Jung Jae Lee ${ }^{5}$, Haewoo Lee ${ }^{6}$, Sung-Doo Won ${ }^{7}$, and Woori Han' \\ ${ }^{1}$ Department of Psychiatry, National Medical Center, Seoul, Republic of Korea \\ ${ }^{2}$ Center for Infectious Diseases, National Medical Center, Seoul, Republic of Korea \\ ${ }^{3}$ Department of Psychiatry, Seoul National University Hospital, Seoul, Republic of Korea \\ ${ }^{4}$ Department of Psychiatry, School of Medicine, Chungnam National University, Daejeon, Republic of Korea \\ ${ }^{5}$ Department of Psychiatry, School of Medicine, Dankook University, Cheonan, Republic of Korea \\ ${ }^{6}$ Department of Psychiatry, Seoul Medical Center, Seoul, Republic of Korea \\ ${ }^{7}$ Department of Clinical Psychology, Keyo Hospital, Uiwang, Republic of Korea
}

Objective The relationship among chronic fatigue, depressive symptoms, and post-traumatic stress symptoms (PTSSs) among Middle East respiratory syndrome (MERS) survivors is poorly understood.

Methods Of 148 survivors who consented to be registered and underwent assessments at 12 months (T1) and 18 months (T2) after the MERS outbreak, 72 (48.65\%) were evaluated for chronic fatigue, depressive symptoms, and PTSSs based on the Impact of Event ScaleRevised (IES-R), the Patient Health Questionnaire-9 (PHQ-9), and the Fatigue Severity Scale (FSS). Data from 52 subjects, who completed both assessments, were analyzed using a regression-based serial multiple mediation model (PROCESS Model 6).

Results Bootstrap analyses indicated no direct effects of T1 FSS on T2 IES-R but significant positive indirect effects of T1 FSS on T2 IESR through T1 PHQ-9 and T2 PHQ-9 (B=2.1601, SE=1.3268, 95\% confidence interval=0.4250-6.1307). In other words, both T1 PHQ-9 and T2 PHQ-9 fully mediated the relationship between T1 FSS and T2 IES.

Conclusion Chronic fatigue 12 months after MERS had indirect effects on prolonged PTSSs 18 months after MERS via persisting depression in MERS survivors. This finding supports the need to promote interventional programs for emerging infectious disease survivors with chronic fatigue to reduce depression and prevent prolonged PTSSs.

Psychiatry Investig 2019;16(1):59-64

Key Words Chronic fatigue, Depression, Emerging infectious diseases, Middle East respiratory syndrome,

Post-traumatic stress symptoms, Survivors.

\section{INTRODUCTION}

A large outbreak of Middle East respiratory syndrome (MERS) infection ${ }^{1}$ occurred in South Korea in 2015, eventually resulting in 186 confirmed cases and 36 deaths. MERS is a lower respiratory tract infection that causes fever, coughing, breathing difficulties, and pneumonia. The condition can progress to acute respiratory distress syndrome and multi-or-

Received: August 16, 2018 Revised: October 6, 2018 Accepted: October 22, 2018

$\triangle$ Correspondence: Hyoung-Shik Shin, MD, PhD

Center for Infectious Diseases, National Medical Center, 245 Eulji-ro, Junggu, Seoul 04564, Republic of Korea

Tel: +82-2-2260-7558, Fax: +82-2-2268-0803, E-mail: hyoungsshin@gmail.com

(a) This is an Open Access article distributed under the terms of the Creative Commons Attribution Non-Commercial License (https://creativecommons.org/licenses/bync/4.0) which permits unrestricted non-commercial use, distribution, and reproduction in any medium, provided the original work is properly cited. gan failure and lead to death in $20-40 \%$ of cases.

Research conducted on emerging infectious diseases (EIDs) in the past decade suggests that psychiatric morbidities and chronic fatigue persisted and continued to be clinically significant among survivors. ${ }^{2,3}$ A study that examined stress levels and psychological distress reported that severe acute respiratory syndrome (SARS) survivors showed high levels of depression, anxiety, and post-traumatic stress symptoms (PTSSs) 1 year after the outbreak. ${ }^{4}$ In a 3-year study, fatigue and psychiatric co-morbidities, including depression, posttraumatic stress disorder (PTSD), somatoform pain disorder, and panic disorder were common in SARS survivors. ${ }^{5}$ A study that evaluated psychological distress and social support of Nigerian patients infected with Ebola virus disease (EVD) reported that survivors experienced an inability to concentrate, lack of sleep, unhappiness, and depression. ${ }^{6}$ Severe depres- 
sion was diagnosed between 1 and 19 months after discharge from an Ebola treatment center among survivors of EVD in Conakry. ${ }^{7}$

Several studies have reported mental health issues in MERSrelated patients. In a study of subjects who had been isolated because they had had contact with a MERS patient, 7.6\% showed anxiety and $16.6 \%$ had developed feelings of anger during the isolation period. ${ }^{8} \mathrm{~A}$ cross-sectional study that examined the mental health of nurses working at a governmentdesignated hospital during a MERS-Coronavirus (CoV) outbreak revealed that the influences of stigma and hardiness on mental health are partially mediated through stress. ${ }^{9}$ Psychological trauma of MERS victims and bereaved families has also been anecdotally reported, ${ }^{10}$ as many patients suffer from rumors and discrimination even after being treated and cleared of the disease.

However, few studies have addressed chronic fatigue and mental health among survivors after a MERS infection in South Korea. Moreover, the relationship between them is unclear, although understanding their relationship is important to identify EID survivors who are at high risk for chronic fatigue and mental health problems and to provide targeted interventions and treatments.

Thus, we investigated symptoms of chronic fatigue, PTSD, and depression in survivors after a MERS epidemic and the relationships among them. A serial multiple mediation model with one independent variable was used. The purpose of this study is to examine the longitudinal mediating effects of depressions between chronic fatigue and later PTSSs for the MERS survivors. Through this, we will reveal predictive factors of PTSSs and we would utilize practical interventions for improving the PTSSs through the development of programs. Furthermore, this study will be utilized as a basis to establish the policy to improve the detrimental mental health effects of the EID infection to survivors.

\section{METHODS}

\section{Participants and procedures}

The study data were obtained from a multi-centered prospective follow-up study of MERS survivors in the Republic of Korea. All survivors with confirmed MERS infection were invited to participate in the study 12 months after the MERS outbreak had ended and follow-up in every 6 months later. Personalized letters and subsequent telephone contact were made to invite subjects to participate, and assessments were arranged.

Of 148 survivors, 72 (48.65\%) consented to be registered. The assessments were performed 12 months (T1) and 18 months (T2) after the MERS outbreak in five hospitals: National Medi- cal Center ( $\mathrm{n}=15)$, Seoul National University Hospital $(\mathrm{n}=17)$, Chungnam National University Hospital ( $\mathrm{n}=13)$, Seoul Medical Center $(\mathrm{n}=12)$, and Dankook University Hospital $(\mathrm{n}=6)$. All participants visited the hospitals for regular health checkups and completed self-report questionnaires. The complete responses from 63 and 54 participants were gathered at T1 and $\mathrm{T} 2$, respectively. Only data from 52 subjects who completed both assessments were analyzed to examine the relationships among the relevant psychological variables.

\section{Variables and measurements}

Data on demographic variables including sex, age, job, and marriage were gathered. Data on medical illnesses prior to MERS and a psychiatric history were also collected. Participants completed several scales, including the Fatigue Severity Scale (FSS), the Patient Health Questionnaire-9 (PHQ-9), and the Impact of Event Scale-Revised (IES-R).

The FSS is a Likert scale consisting of nine items that assess fatigue severity and functionality during the past one week. ${ }^{11}$ Items are rated on a scale of 1 to 7 according to their level of agreement with a given statement, and include such statements as "I am easily fatigued" or "fatigue interferes with carrying out certain duties and responsibilities." Values for each item were averaged for a composite score, with higher scores indicating higher levels of impairment from fatigue and fatigue was largely independent of self-reported depressive symptoms. ${ }^{11}$ Using the Korean FSS ${ }^{12}$ index of 3.22 as the cutoff point, sensitivity was $84.1 \%$ and specificity was $85.7 \%$ for the fatigue and control groups, respectively. Factor analysis showed first factor was recognition of fatigue and second factor was the interference of fatigue on functioning in physical, family and social area. ${ }^{12}$

The PHQ- $9^{13}$ is used to monitor depression severity. The questionnaire consists of nine items based on the Diagnostic and Statistical Manual of Mental Disorders, fourth edition criteria for depression, scored from 0 to 3 , providing a severity score ranging from 0 to 27 . Accordingly, depression severity was defined as no depression (1-4), mild depression (5-9), moderate depression (10-14), moderately severe depression (15-19), and severe depression (20-27). The Korean version of the PHQ-9 is a reliable and valid tool for screening and assessing depressed patients (Cronbach's alpha coefficient $=0.81$ ). ${ }^{14}$

The IES-R was designed to assess symptoms associated with PTSD following a traumatic life event (MERS infection in our study). Symptoms are measured on 22 self-reported items for a total subjective stress assessment that can be further divided into intrusion, avoidance, and hyperarousal subscales. Individual items describe difficulties associated with stressful events, and participants were asked to indicate the extent to which each item distressed or bothered them over 
the last 7 days using a rating scale of $0-4$. A total score $\geq 25$ is considered clinically significant. ${ }^{15}$

\section{Statistical analyses}

All statistical analyses were performed using the Statistical Package for Social Science 18.0 software (SPSS Inc., Chicago, IL, USA). We performed Paired T-test to examine significant changes in all mean scores over the time period. Pearson's correlation analyses were used to determine the relationships among chronic fatigue (T1 FSS), depression (T1 PHQ, T2 PHQ), and PTSS (T2 IES). The hypothesis that chronic fatigue affects PTSS through depression was examined using the serial multiple mediation model, which is a kind of multiple regression in the SPSS PROCESS macro. ${ }^{16,17}$ Serial multiple-mediated-effects analyses were conducted with 5,000 bootstrap- ping repetitions. Confidence intervals that did not include 0 were considered significant. All tests were two-sided. A pvalue $<0.05$ was considered significant.

\section{Ethics statement}

The present study protocol was reviewed and approved by the Institutional Review Board of the National Medical Center (IRB No. H-1510-059-007). Informed consent was submitted by all subjects when they were enrolled. All participants were informed that they could withdraw from the study at any time.

\section{RESULTS}

Table 1 presents the demographic and clinical characteris-

Table 1. Demographic and clinical characteristics of participants

\begin{tabular}{|c|c|c|c|}
\hline \multirow{2}{*}{ Variables } & \multirow{2}{*}{ Subcategory } & \multicolumn{2}{|c|}{ Total $(\mathrm{N}=52)$} \\
\hline & & $\mathrm{N}$ or mean & $\%$ or $\mathrm{SD}$ \\
\hline \multirow[t]{2}{*}{ Gender } & Male & 32 & 61.5 \\
\hline & Female & 20 & 38.5 \\
\hline Age & & 49.7 & 12.0 \\
\hline \multirow[t]{3}{*}{ Job } & Employed & 30 & 57.7 \\
\hline & Unemployed or retired & 9 & 17.3 \\
\hline & Others (housewife, self-employed, etc.) & 13 & 25.0 \\
\hline \multirow[t]{3}{*}{ Marriage } & Married & 43 & 82.7 \\
\hline & Unmarried & 5 & 9.6 \\
\hline & Divorce or bereaved & 4 & 7.7 \\
\hline Medical illness prior to MERS & Yes & 16 & 30.8 \\
\hline \multirow[t]{2}{*}{ Previous history of psychiatric visit } & Yes & 7 & 13.5 \\
\hline & & $\mathrm{N}$ or median & $\%$ or $\mathrm{SD}$ \\
\hline \multirow[t]{5}{*}{ Status at the point of infection } & Patients & 16 & 30.8 \\
\hline & Health care workers & 12 & 23.1 \\
\hline & Caregivers & 10 & 19.2 \\
\hline & Visitors & 9 & 17.3 \\
\hline & Others & 5 & 9.6 \\
\hline Days of hospitalization & & 21 & 21.8 \\
\hline Days from symptoms to confirmed diagnosis & & 4.5 & 4.1 \\
\hline Total days of illness & & 23 & 27.1 \\
\hline Pneumonia & Yes & 19 & 36.5 \\
\hline Ventilator treatment & Yes & 10 & 19.2 \\
\hline \multirow[t]{2}{*}{ ECMO treatment } & Yes & 2 & 3.8 \\
\hline & Clinical cut off & $\mathrm{T} 1$ & $\mathrm{~T} 2$ \\
\hline PHQ-9 & $\geq 10.0$ & $14(26.9)$ & $9(17.3)$ \\
\hline FSS & $\geq 3.22$ & $25(48.1)$ & $17(32.7)$ \\
\hline IES-R & $\geq 25.0$ & $22(42.3)$ & $14(26.9)$ \\
\hline
\end{tabular}

FSS: Fatigue Severity Scale, PHQ-9: Patient Health Questionnaire-9, IES-R: Impact of Event Scale-Revised, T1: 12 months after MERS, T2: 18 months after MERS. MERS: Middle East respiratory syndrome, ECMO: extracorporeal membrane oxygenation, SD: standard deviation 
tics of the participants. Of the 52 participants included in the study, 32 (61.54\%) were male and 20 (38.46\%) were female. The mean age of the participants was about 50 years (mean= $49.69, \mathrm{SD}=11.97)$. More than half $(\mathrm{n}=30,57.69 \%)$ of the participants were employed, and $82.69 \%(n=43)$ were married. Nearly one-third ( $30.77 \%, \mathrm{n}=16)$ had suffered from other medical illnesses before contracting MERS and $13.46 \%(n=7)$ had received previous psychiatric care before contracting MERS.

In all, 23.08\% of cases were healthcare workers when they were infected with MERS. The mean number of hospitalization days was $21.00(\mathrm{SD}=21.76)$. The mean number of days from symptoms to the confirmed diagnosis was $4.50(\mathrm{SD}=4.12)$, and the mean total days of illness was 23.00 ( $\mathrm{SD}=27.11)$. About $36.54 \%(n=19)$ of participants had suffered from pneumonia, $19.23 \%(n=10)$ had been treated with a ventilator, and 3.85\% $(n=2)$ had been treated with extracorporeal membrane oxygenation during the acute MERS treatment period.

Changes in the number of subjects with clinically relevant symptoms over time are shown in Table 1. The PHQ-9 scores indicated that $26.92 \%$ (T1), $17.31 \%$ (T2) of subjects had clinically relevant depressive symptoms. In addition, $48.08 \%$ (T1), 32.69\% (T2) had clinically relevant chronic fatigue according to their FSS scores. In terms of the IES-R assessment, $42.31 \%$ (T1), 26.92\% (T2) had scores above the cut-off point for PTSD.

Table 2 shows the correlations among the variables of interest included in the hypothetical model. All correlations across the variables of interest were significant. FSS (T1) scores were positively correlated with scores on the PHQ-9 $(\mathrm{T} 1, \mathrm{~T} 2)$ and the IES-R (T2). Mean scores of all of the scales were lower at T2 than at T1. The mean FSS score changed significantly from $3.50(\mathrm{SD}=1.88)$ to $2.78(\mathrm{SD}=1.89)$, and that of the IES-R decreased significantly from 25.83 ( $\mathrm{SD}=20.05$ ) to 19.29 ( $\mathrm{SD}=21.03)$. The mean score of the PHQ-9 decreased from $6.50(\mathrm{SD}=5.69)$ to $5.42(\mathrm{SD}=5.71)$ but we could not find significance.

The serial multiple-mediation model, which included T1 FSS, T1 PHQ-9, and T2 PHQ-9, accounted for $34.19 \%$ of the

Table 2. Descriptive data and correlations among variables $(\mathrm{N}=52)$

\begin{tabular}{|c|c|c|c|c|c|c|c|c|}
\hline Variables & $\mathrm{M}$ & SD & 1 & 2 & 3 & 4 & 5 & 6 \\
\hline 1. FSS & 3.50 & 1.88 & - & & & & & \\
\hline 2. FSS & 2.78 & 1.89 & $0.677^{* * *}$ & - & & & & \\
\hline 3. PHQ-9 T1 & 6.50 & 5.69 & $0.742^{* * *}$ & $0.579^{* * *}$ & - & & & \\
\hline 4. PHQ-9 T2 & 5.42 & 5.71 & $0.630^{* * *}$ & $0.698^{* * *}$ & $0.712^{* * *}$ & - & & \\
\hline 5. IES-R T1 & 25.83 & 20.05 & $0.712^{* * *}$ & $0.700^{* * *}$ & $0.768^{* * *}$ & $0.677^{* * *}$ & - & \\
\hline 6. IES-R T2 & 19.29 & 21.03 & $0.577^{* * *}$ & $0.765^{* * *}$ & $0.617^{* * *}$ & $0.688^{* * *}$ & $0.787^{* * *}$ & - \\
\hline
\end{tabular}

*** $\mathrm{p}<0.001$. FSS: Fatigue Severity Scale, PHQ: Patient Health Questionnaire-9, IES: Impact of Event Scale, T1: 12 months after MERS, T2: 18 months after MERS. MERS: Middle East respiratory syndrome, SD: standard deviation

Table 3. The Bootstrap results for the indirect effect of 12 months severe fatigue on 18 months impact of traumatic event between 12 and 18 months depression

\begin{tabular}{|c|c|c|c|c|}
\hline & \multirow{2}{*}{$\mathrm{B}$} & \multirow{2}{*}{ SE } & \multicolumn{2}{|c|}{$95 \% \mathrm{CI}(\mathrm{BC})$} \\
\hline & & & Lower & Upper \\
\hline \multicolumn{5}{|l|}{ Direct effects } \\
\hline FSS T1 $\rightarrow$ PHQ T1 & $2.2628^{* * *}$ & 0.2807 & 1.6991 & 2.8264 \\
\hline PHQ T1 $\rightarrow$ PHQ T2 & $0.5478^{* * *}$ & 0.1448 & 0.2569 & 0.8387 \\
\hline FSS T1 $\rightarrow$ PHQ T2 & 0.6871 & 0.4379 & -0.1924 & 1.5667 \\
\hline PHQ T1 $\rightarrow$ IES T2 & 0.6060 & 0.6205 & -0.6411 & 1.8530 \\
\hline PHQ T2 $\rightarrow$ IES T2 & $1.7427^{* *}$ & 0.5342 & 0.6690 & 2.8163 \\
\hline FSS T1 $\rightarrow$ IES T2 & 1.7808 & 1.6945 & -1.6244 & 5.1860 \\
\hline \multicolumn{5}{|l|}{ Indirect effects } \\
\hline Path 1: FSS T1 $\rightarrow$ PHQ T1 $\rightarrow$ IES T2 & $1.3711^{\mathrm{b}}$ & 1.6396 & -1.8440 & 4.6540 \\
\hline Path 2: FSS T1 $\rightarrow$ PHQ T1 $\rightarrow$ PHQ T2 $\rightarrow$ IES T2 & $2.1601^{\mathrm{a}}$ & 1.3268 & 0.4250 & 6.1307 \\
\hline Path3: FSS T1 $\rightarrow$ PHQ T2 $\rightarrow$ IES T2 & $1.1974^{\mathrm{b}}$ & 0.8451 & 0.1141 & 3.5656 \\
\hline
\end{tabular}

${ }^{* *} \mathrm{p}<0.01,{ }^{* * *} \mathrm{p}<0.001$. CI (BC)=bias corrected confidence interval; ${ }^{\mathrm{a}}=$ significant; ${ }^{\mathrm{b}}=$ not significant. FSS: Fatigue Severity Scale, PHQ: Patient Health Questionnaire-9, IES: Impact of Event Scale, T1: 12 months after MERS, T2: 18 months after MERS. MERS: Middle East respiratory syndrome 


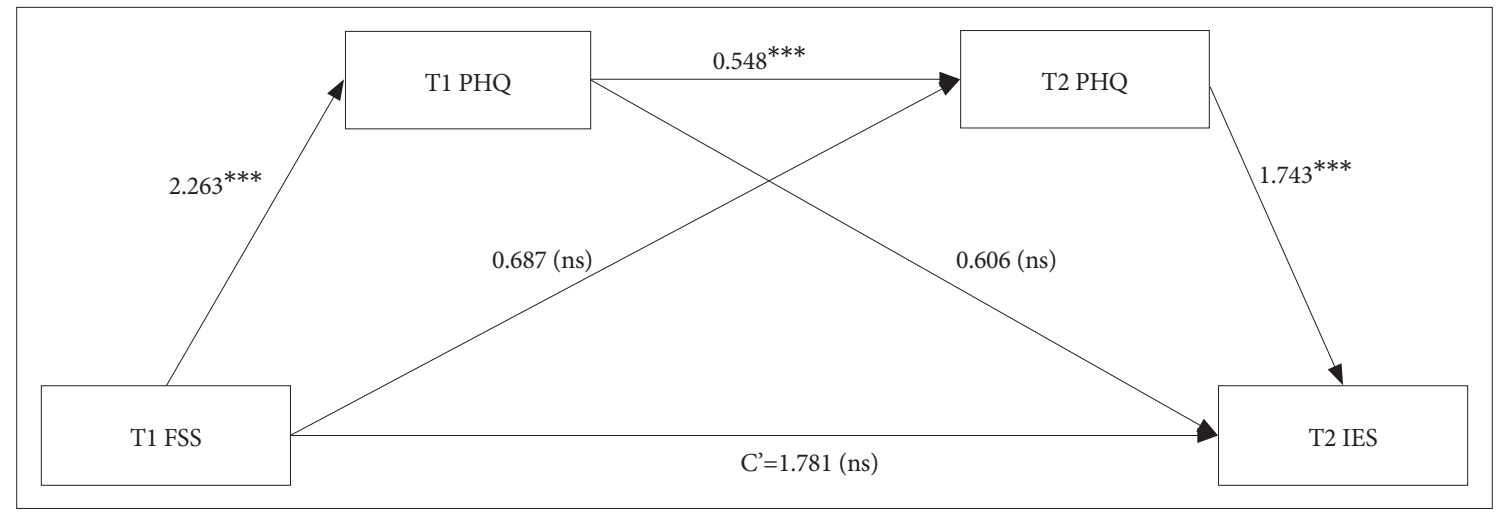

Figure 1. Model depicting the effect of severe fatigue, depression on post-trauma of MERS. ${ }^{* *} p<0.01,{ }^{* * *} p<0.001$. FSS: Fatigue Severity Scale, PHQ: Patient Health Quetionnaire-9, IES: Impact of Event Scale, T1: 12 months after MERS, T2: 18 months after MERS, c': direct effect of $X$ on $Y(X=T 1 F S S ; Y=T 2 ~ I E S)$, ns: not significant. MERS: Middle East respiratory syndrome.

variance in T2 IES-R $\left[\mathrm{R}^{2}=0.3419, \mathrm{~F}(1,51)=26.4934, \mathrm{p}<0.001\right]$. As shown in Table 3, T1 FSS was associated with T1 PHQ-9 $(\mathrm{B}=2.2628, \mathrm{SE}=0.2807, \mathrm{p}<0.001)$ but not with T2 IES-R $(\mathrm{B}=$ 1.7808, $\mathrm{SE}=1.6945, \mathrm{p}=0.2984)$ or T2 PHQ-9 $(\mathrm{B}=0.6871, \mathrm{SE}=$ $0.4379, \mathrm{p}=0.1229)$. Moreover, T1 PHQ-9 was associated with T2 PHQ-9 $(B=0.5478, \mathrm{SE}=0.1448, \mathrm{p}<0.001)$ but not with T2 IES-R $(B=0.6060, S E=0.6205, p=0.3336)$. On the other hand, T2 PHQ- 9 was associated with T2 IES-R $(B=1.7427, \mathrm{SE}=$ $0.5342, \mathrm{p}<0.01$ ).

Moreover, bootstrap analyses revealed that the positive indirect effects of T1 FSS on T2 IES-R through T1 PHQ-9 and T2 PHQ-9 were significant $(\mathrm{B}=2.1601, \mathrm{SE}=1.3268$, $95 \%$ confidence interval $=0.4250-6.1307$ ) (Table 3). These results indicate that both T1 PHQ and T2 PHQ-9 fully mediated the effects of T1 FSS on T2 IES-R (Figure 1), which suggests that both T1 PHQ and T2 PHQ-9 eliminated the direct effects of T1 FSS on T2 IES-R.

\section{DISCUSSION}

Chronic fatigue 12 months post-MERS had indirect effects on prolonged PTSSs 18 months post-MERS via persisting depression in MERS survivors. This is in agreement with a longitudinal study ${ }^{18}$ of anxiety, depression, and fatigue in patients with multiple sclerosis that showed that depression strongly predicts anxiety and fatigue, and that anxiety and fatigue strongly predict subsequent depression. Moreover, previous studies conducted on subjects who had experienced disasters noted that current depression is a predictor of PTSSs. ${ }^{19}$

The rate of participants reported clinically significant symptoms in CFS (32.7-48.1\%), depression (17.3-26.9\%), and PTSD (26.9-42.3\%) was comparable to that following SARS. ${ }^{4}$ In a 4 -year survey in Hong Kong, more than $40 \%$ of the respondents had active psychiatric illness, and $40.3 \%$ reported a chronic fatigue problem. ${ }^{20}$ In addition, a cohort study that investigated psychiatric complications among SARS survivors 30 months after infection found that the prevalence of any psychiatric disorder was $33.3 \% ; 25.6 \%$ of patients had PTSD and $15.6 \%$ had depressive disorders. ${ }^{21}$ Moreover, MERS survivors shared similar psychopathologies as other disaster survivors ${ }^{22}$ who reported varying rates of PTSD $\left.(30-40 \%)\right)^{23}$ depression (25\%), and fatigue symptoms (50\%). These findings suggest that EIDs result not only in physical problems but also psychiatric sequelae in infected persons because they might experience those as disastrous events.

Fatigue is a prominent disabling symptom in a variety of medical and neurologic disorders. In EIDs survivors, the Coronavirus itself may have directly caused sleep disorders, fatigue, and behavioral symptoms, which in turn may have resulted in chronic post-inflammatory central nervous system pathologies that adversely affected sleep, pain sensitivity, and energy. It is noteworthy that chronic fatigue syndrome (CFS) shares many symptoms with depression and the two are frequently co-diagnosed..$^{24}$ However, according to the study which measure the fatigue severity among patients with multiple sclerosis, patients with systemic lupus erythematosus, and healthy adults, fatigue was largely independent of self-reported depressive symptoms. ${ }^{11}$ Despite this, the current study appears to be the only longitudinal examination of chronic fatigue, depression, and PTSSs in EIDs survivors.

We found that the severities of chronic fatigue and PTSS improved significantly but that of depression relatively unchanged throughout the study period. Although this was the short-term follow-up study, these results suggest we should give attention to the persisting depression during recovery period in MERS survivors. It would be important for clinicians to assess depression among EIDs survivors with CFS. Furthermore our findings suggest the need to promote interventional programs for MERS survivors with chronic fatigue to prevent prolonged PTSSs by ameliorate depression. Two pos- 
sible treatments for chronic fatigue syndrome (CFS) are cognitive behavioral therapy and graded exercise therapy. ${ }^{25}$ There is less clear evidence of the benefit of drug therapy for CFS in patients without comorbid depression or anxiety disorders.

There were several limitations to this study. First, some sampling bias may have occurred, as $<50 \%$ of the MERS survivors in Korea consented to participate in the study. It is possible that people who were heavily affected by MERS or who were experiencing mental health difficulties were more prone to participate in the research. In addition, mental health and fatigue symptoms were evaluated using self-reported measures that are not diagnostic in nature but result in assessments of symptomatology. However, these measures have demonstrated validity and reliability in Korea and are consistently correlated with more comprehensive diagnostic tools used to assess corresponding symptoms. Despite these limitations, the present study is the first to examine the relationships among chronic fatigue, depression, and PTSSs in survivors after the MERS epidemic in Korea.

In summary, this longitudinal study revealed that many MERS survivors suffered from chronic fatigue and mental health problems even more than 1 year after the outbreak in South Korea, although three scores showed a decreasing trend. The prevalence rates of CFS, depression, and PTSD were $32.7 \%, 17.3 \%$, and $26.9 \%$, respectively, 18 months after the MERS outbreak. Furthermore, chronic fatigue 12 months postMERS had an indirect effect on prolonged PTSS 18 months post-MERS through depression among survivors. Our findings may inform future strategies for detecting survivors who are at high risk for PTSD and for promoting long-term mental health outcomes in the event of other EIDs.

\section{Acknowledgments}

This research was supported by a grant of the Korea Health Technology R\&D Project through the Korea Health Industry Development Institute (KHIDI), funded by the Ministry of Health \& Welfare, Republic of Korea (HI15C3227) and a grant from the Korean Mental Health Technology R\&D Project, Ministry of Health \& Welfare, Republic of Korea (HM15C1054).

\section{REFERENCES}

1. Cho SY, Kang JM, Ha YE, Park GE, Lee JY, Ko JH, et al. MERS-CoV outbreak following a single patient exposure in an emergency room in South Korea: an epidemiological outbreak study. Lancet 2016;388:9941001.

2. Moldofsky H, Patcai J. Chronic widespread musculoskeletal pain, fatigue, depression and disordered sleep in chronic post-SARS syndrome; a case-controlled study. BMC Neurol 2011;11:37.

3. Tansey CM, Louie M, Loeb M, Gold WL, Muller MP, de Jager J, et al. One-year outcomes and health care utilization in survivors of severe acute respiratory syndrome. Arch Intern Med 2007;167:1312-1320.

4. Lee AM, Wong JG, McAlonan GM, Cheung V, Cheung C, Sham PC, et al. Stress and psychological distress among SARS survivors 1 year after the outbreak. Can J Psychiatry 2007;52:233-240.
5. Wing YK, Leung CM. Mental health impact of severe acute respiratory syndrome: a prospective study. Hong Kong Med J 2012;18:24-27.

6. Mohammed A, Sheikh TL, Gidado S, Poggensee G, Nguku P, Olayinka A, et al. An evaluation of psychological distress and social support of survivors and contacts of Ebola virus disease infection and their relatives in Lagos, Nigeria: a cross sectional study-- 2014. BMC Public Health 2015;15:1-8.

7. Keita MM, Taverne B, Sy Savané S, March L, Doukoure M, Sow MS, et al. Depressive symptoms among survivors of Ebola virus disease in Conakry (Guinea): preliminary results of the PostEboGui cohort. BMC Psychiatry 2017;17:127.

8. Jeong H, Yim HW, Song YJ, Ki M, Min JA, Cho J, et al. Mental health status of people isolated due to Middle East Respiratory Syndrome. Epidemiol Health 2016;38:e2016048.

9. Park JS, Lee EH, Park NR, Choi YH. Mental health of nurses working at a government-designated hospital during a MERS-CoV outbreak: a cross-sectional study. Arch Psychiatr Nurs 2018;32:2-6.

10. Sim M. Psychological trauma of Middle East Respiratory Syndrome victims and bereaved families. Epidemiol Health 2016;38:e2016054.

11. Krupp LB, LaRocca NG, Muir-Nash J, Steinberg AD. The fatigue severity scale: application to patients with multiple sclerosis and systemic lupus erythematosus. Arch Neurol 1989;46:1121-1123.

12. Chung KI, Song CH. Clinical usefulness of fatigue severity scale for patients with fatigue, and anxiety or depression. Korean J Psychosom Med 2001;9:164-173.

13. Kroenke K, Spitzer RL, Williams JB. The PHQ-9: validity of a brief depression severity measure. J Gen Intern Med 2001;16:606-613.

14. Kroenke K, Spitzer RL. The PHQ-9: a new depression diagnostic and severity measure. Psychiatr Ann 2002;32:509-515.

15. Eun HJ, Kwon TW, Lee SM, Kim TH, Choi MR, Cho SJ. A study on reliability and validity of the Korean version of impact of event scale-revised. J Korean Neuropsychiatr Assoc 2005;44:303-310.

16. Hayes AF. PROCESS: a versatile computational tool for observed variable mediation, moderation, and conditional process modeling (White paper). 2012. Available at: http://www.afhayes.com/public/process 2012. pdf. Accessed January 16, 2018.

17. Hayes AF. Introduction to Mediation, Moderation, and Conditional Process Analysis: a Regression-Based Approach. New York, NY: The Guilford Press; 2013.

18. Brown RF, Valpiani EM, Tennant CC, Dunn SM, Sharrock M, Hodgkinson $\mathrm{S}$, et al. Longitudinal assessment of anxiety, depression, and fatigue in people with multiple sclerosis. Psychol Psychother 2009;82:41-56.

19. Chang CM, Connor KM, Lai TJ, Lee LC, Davidson JR. Predictors of posttraumatic outcomes following the 1999 Taiwan earthquake. J Nerv Ment Dis 2005;193:40-46.

20. Lam MH, Wing YK, Yu MW, Leung CM, Ma RC, Kong AP, et al. Mental morbidities and chronic fatigue in severe acute respiratory syndrome survivors: long-term follow-up. Arch Intern Med 2009;169:21422147.

21. Mak IWC, Chu CM, Pan PC, Yiu MGC, Chan VL. Long-term psychiatric morbidities among SARS survivors. Gen Hosp Psychiatry 2009;31: 318-326.

22. Velden PGV, Grievink L, Kleber RJ, Drogendijk AN, Roskam AJ, Marcelissen FG, et al. Post-disaster mental health problems and the utilization of mental health services: a four-year longitudinal comparative study. Adm Policy Ment Health 2006;33:279-288.

23. Neria Y, Nandi A, Galea S. Post-traumatic stress disorder following disasters: a systematic review. Psychol Med 2008;38:467-480.

24. Fuller-Thomson E, Nimigon J. Factors associated with depression among individuals with chronic fatigue syndrome: findings from a nationally representative survey. Fam Pract 2008;25:414-422.

25. Yancey JR, Thomas SM. Chronic fatigue syndrome: diagnosis and treatment. Am Fam Physician 2012;86:741-746. 